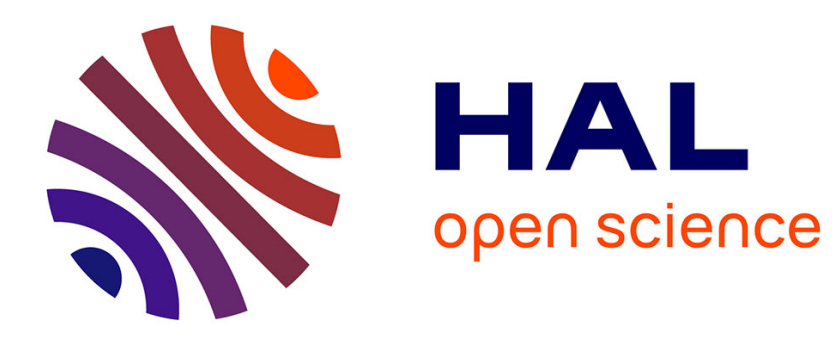

\title{
Blind identification of MISO-FIR channels
}

Carlos Estêvão Rolim Fernandes, Pierre Comon, Gérard Favier

\section{To cite this version:}

Carlos Estêvão Rolim Fernandes, Pierre Comon, Gérard Favier. Blind identification of MISO-FIR channels. Signal Processing, 2010, 90, pp.490-503. 10.1016/j.sigpro.2009.07.023 . hal-00420044

\section{HAL Id: hal-00420044 https://hal.science/hal-00420044}

Submitted on 27 Sep 2009

HAL is a multi-disciplinary open access archive for the deposit and dissemination of scientific research documents, whether they are published or not. The documents may come from teaching and research institutions in France or abroad, or from public or private research centers.
L'archive ouverte pluridisciplinaire HAL, est destinée au dépôt et à la diffusion de documents scientifiques de niveau recherche, publiés ou non, émanant des établissements d'enseignement et de recherche français ou étrangers, des laboratoires publics ou privés. 


\title{
Blind identification of MISO-FIR channels
}

\author{
Carlos Estêvão R. Fernandes ${ }^{\dagger *}$, Pierre Comon $^{\ddagger}$, Gérard Favier ${ }^{\ddagger}$ \\ Emails: estevao@ufc.br, pcomon@i3s.unice.fr, favier@i3s.unice.fr \\ ${ }^{\dagger}$ Federal University of Ceará, Computer Engineering Department \\ Campus UFC, Praça Sen. Figueira, rua Anahid Andrade s/n, Sobral-CE, Brazil. \\ ${ }^{\ddagger}$ I3S Laboratory, University of Nice Sophia Antipolis, CNRS, \\ 2000 route des Lucioles, 06903, Sophia Antipolis, France. \\ Phone: +33492942736 Fax: +33492942896.
}

To appear in Signal Processing, Elsevier, vol.90

\begin{abstract}
In this paper, we address the problem of determining the order of MISO channels by means of a series of hypothesis tests based on scalar statistics. Using estimated 4th-order output cumulants, we exploit the sensitiveness of a Chi-square test statistic to the non Gaussianity of a stochastic process. This property enables us to detect the order of a linear finite impulse response (FIR) channel. Our approach leads to a new channel order detection method and we provide a performance analysis along with a criterion to establish a decision threshold, according to a desired level of tolerance to false alarm. Afterwards, we introduce the concept of MISO channel nested detectors based on a deflation-type procedure using the 4th-order output cumulants. The nested detector is combined with an estimation algorithm to select the order and estimate the parameters associated with different transmitters composing the MISO channel. By treating successively shorter and shorter channels, it is also possible to determine the number of sources.
\end{abstract}

Index terms: Blind channel parameter estimation, channel order detection, Chi-square test, highorder statistics, MISO channels.

\footnotetext{
*This work has been developed under the CAPES/COFECUB Ma 544/07 Research contract and partially supported by the PNPD Program of the CAPES agency in collaboration with the Teleinformatics Engineering Dept. of the Federal University of Ceará, Fortaleza, Brazil.
} 


\section{Introduction}

Convolutive propagation channels are typical in wireless, satellite and radiocommunication systems. The channel memory is known to be closely related to the delay spread profile produced by the multipath propagation scenario and it indicates the length of the channel impulse response. Long delay spread profiles characterize highly frequency-selective channels and introduce intersymbol interference (ISI) in the sampling process. The order of the radio mobile channel relates the length of the channel impulse response with the symbol period by indicating the number of past symbols being convolved with the current one. In practice, the channel order is given by the number of symbol periods fitting the (truncated) channel delay spread profile. Most channel parameter estimation algorithms require the knowledge of the channel order or, at least an upper bound of it 31. Channel order mismatches may have very costly consequences, including bit error rate (BER) floors, signal-to-noise ratio (SNR) penalties and numerical instabilities [28, 29, 18, 11].

In this paper, we address the problem of determining the order of finite impulse response (FIR) channels in the context of a multiple-input single-output (MISO) communication system, using only the 4th-order cumulants of the received signal. Channel order estimation is a classic model selection problem strongly related to determining the number of signals embedded in noisy observations in narrow-band array processing. This has been often referred to as the signal (or source) detection problem [1, 11, 10]. Classical procedures for model order determination are based on multiple hypothesis testing and make use of the eigenvalues of a sample correlation matrix. This is the case of the well-known sphericity test [32, 30], which estimates the number of model parameters by testing on adjacent groups of eigenvalues, in the case of a Gaussian process [37]. This approach has been widely used for solving the signal detection problem in the context of passive arrays [50, 51]. Other traditional techniques are based on information theoretic criteria that minimize the information lost due to the model approximation 44. 47. The Akaike's Information Criterion (AIC) [1] as well as the Rissanen's Minimum Description Length (MDL) [42], also known as the Schwarz Bayesian Criterion (SBC) 43, can both be used to test the equality of the smallest eigenvalues of the sample correlation matrix. Since AIC is not consistent [43], it tends to overestimate the model order, even for high SNR values [25]. In general, MDL performs better than AIC, but it tends to an underestimation at low and medium SNR [26]. Similar results have also been demonstrated in the context of source detection in passive arrays [48, 49].

Unlike previous works, the order detection method proposed in this paper is based on a scalar Chisquare test statistic that is sensitive to the non Gaussianity of a stochastic process, since it is derived from the multivariate estimator of the 4th-order output cumulants. Exploiting this property enables us to detect the order of a single-input single-output (SISO) communication channel. Making use of existing results on the asymptotic variance of the test variable, we present a performance analysis of the proposed detector. In addition, we establish a criterion for fixing the decision threshold based on a given level of tolerance to false alarm. Some implementations of the proposed method are provided in the context of radiocommunication channels demonstrating very good fit between the empirical and the theoretical results.

In the case of MISO communication channels, the propagation scenario can be viewed as a highly underdetermined convolutive mixture (more sources than sensors). Overdetermined mixtures have been exhaustively studied in the literature, including instantaneous [4, 8, 12] as well as convolutive mixtures (c.f. 33, 34, 35, 36, 45, 46] and references therein). The case of underdetermined mixtures, on the other hand, has only recently been treated [3, 16, 17, 21, 52], and systems with one single output sensor have received considerably less attention. In this work, we introduce a deflation-type approach to solve the 
MISO channel order detection and identification problem. The proposed method is based on the use of a combined procedure that jointly selects the order, detects the number of sources and estimates the parameters of the MISO channel. The so-called HOS-based nested detector searches for the longest channel, determines its order and estimates its coefficients. Then, based on the deflation principle, it successively tests and detects the presence of shorter and shorter channels. Our detector makes use of a Chi-square test for the channel order selection and of blind identification techniques for estimating the MISO channel coefficients using 4th-order cumulants. A preliminary version of this deflation approach was introduced in [20], where only the case of $2 \times 1$ MISO systems has been considered and the order detection test statistics were based on the eigenvalues of 4 th-order output cumulant matrices.

The main contributions of this work can be summarized as follows: $i$.) determination of a Chi-square test statistic based on 4th-order output cumulants; ii.) introduction of a blind method for determining the order of a SISO channel; iii.) proposition of a blind channel identification method based on a rank-one approximation of a 4th-order cumulant matrix; $i v$.) development of a combined blind procedure for order detection and channel identification in the context of MISO channels.

The rest of this paper is organized as follows: in section 2, we derive a Chi-square test statistic based on 4th-order output cumulant information; the principles underlying our SISO channel order detector are introduced and an asymptotic performance analysis is carried out. Then, in section 3 , we introduce the concept of nested detectors for combined order detection and blind identification in the context of convolutive MISO communication channels; this idea exploits the residual 4th-order information remaining after subtraction of the reconstructed cumulants of previously estimated sources. In section 6 , computer simulation results are provided to illustrate the performance obtained in terms of channel order detection and identification. We finally draw our conclusions in section 司 along with some perspectives for future works.

\section{HOS-based Chi-square test for SISO channel order detection}

In order to introduce the main ideas behind our channel order detector, we first consider the case of a single source and a single receive antenna. In section 2.1, we present the SISO channel model and the associated assumptions. Then, we define the 4th-order output cumulants and give the expressions for the (circular and noncircular) covariance matrices of the cumulant estimators, which are important for the calculation of the Chi-Square test variable, introduced in section 2.2. The SISO channel order detection algorithm is presented in section 2.3, under the form of a hypothesis test. A performance analysis of the test is provided and the determination of the decision threshold is addressed making use of the probability distribution function of the test variable.

\subsection{Channel model and 4th-order output cumulants}

Let us consider the baseband representation of a radiocommunication channel in which the output signal $y(n)$, after sampling at the symbol rate, is written as follows:

$$
\begin{gathered}
x(n)=\sum_{\ell=0}^{L} h(\ell) s(n-\ell), \\
y(n)=x(n)+v(n),
\end{gathered}
$$

where $h(0)=1$ and the complex coefficients $h(\ell)$ represent the equivalent discrete impulse response of the channel, including pulse shaping and receiving filters. Throughout the rest of this work, we denote 
the channel order by $L$, and the superscripts $(\cdot)^{*},(\cdot)^{\top}$ and $(\cdot)^{\mathrm{H}}$ denote the complex-conjugate, matrix transpose and matrix conjugate-transpose (Hermitian), respectively. The following assumptions hold:

A1 : The non-observable discrete input sequence $s(n)$ is complex-valued, ergodic, stationary, independent and identically distributed (iid) with symmetric distribution, zero-mean, unit variance $\left(\mathbb{E}\left\{|s(n)|^{2}\right\}=1\right)$ and nonzero Kurtosis given by:

$$
\gamma_{4, s}=\mathbb{E}\left\{|s(n)|^{4}\right\}-\left|\mathbb{E}\left\{s(n)^{2}\right\}\right|^{2}-2,
$$

where $\mathbb{E}\{\cdot\}$ denotes the expectation operator.

A2 : The additive noise sequence $v(n)$ is normally distributed with zero-mean and unknown autocorrelation function. It is assumed to be independent from $s(n)$.

A3 : The FIR filter representing the channel is assumed to be causal with memory $L+1$, i.e. $h(\ell)=0$, $\forall \ell \notin[0, L]$, and $h(\ell) \neq 0$ for $\ell=L$ and $\ell=0$.

A4 : The channel order is bounded by a known value $K$, i.e. $K>L$.

The 4th-order output cumulants are defined as follows (see [5] for a definition of cumulant):

$$
c_{4, y}(i, j, k) \triangleq \operatorname{cum}\left[y^{*}(n), y(n+i), y^{*}(n+j), y(n+k)\right] .
$$

Using the channel model (1), taking assumptions A1 and A2 into account and making use of the multilinearity property of cumulants, we get [6, 过:

$$
c_{4, y}(i, j, k)=\gamma_{4, s} \sum_{\ell=0}^{L} h^{*}(\ell) h(\ell+i) h^{*}(\ell+j) h(\ell+k)
$$

where $\gamma_{4, s}$, defined in A1, is nothing else but $c_{4, s}(0,0,0)$. Let us define the 4th-order output cumulant vectors $\mathbf{c}_{k} \in \mathbb{C}^{P \times 1}$, for $k \in[1, K]$, with elements given by

$$
\left[\mathbf{c}_{k}\right]_{p}=C_{k}^{(p)}, \quad p \in[1, P]
$$

where

$$
C_{k}^{(p)} \triangleq c_{4, y}\left(i_{p}-1, j_{p}-1, k-1\right), \quad\left(i_{p}, j_{p}\right) \in \mathcal{J},
$$

and each pair $\left(i_{p}, j_{p}\right)$ is formed of strictly positive integer numbers, with $i_{p}, j_{p} \leq K$, belonging to the index set

$$
\mathcal{J}=\left\{\left(i_{1}, j_{1}\right) ; \ldots ;\left(i_{P}, j_{P}\right)\right\},
$$

with cardinality $P$. The choice of the index set $\mathcal{J}$ is postponed to section 3.1, in the context of the blind identification of MISO channels. Due to (田) and assumption A3, we have:

$$
C_{k}^{(p)}=0, \forall i_{p}, j_{p}, k>L+1 .
$$

Hence, the 4 th-order output cumulants are zero whenever either $i_{p}, j_{p}$, or $k$ are larger than the channel memory $L+1$. Furthermore, from the above definitions, we also note that $\mathbf{c}_{k}$ can include some purely real-valued components. More precisely, we have:

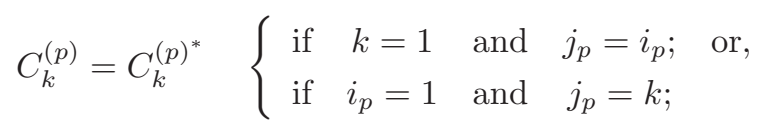


In most of the real-life situations, the true values of the output cumulants are not available and have to be estimated from the output signal samples $y(n), n=0, \ldots, N-1$. Due to the ergodicity assumption, the 2nd- and 4th-order moments can be estimated by means of time averages. The cumulant estimators obtained using the ergodic moment estimates are asymptotically unbiased [5, 27]. The cumulant estimators are also consistent, since their variance is asymptotically zero.

We define the complex-valued estimator $\hat{\mathbf{c}}_{k}$ as:

$$
\hat{\mathbf{c}}_{k}=\left[\begin{array}{lll}
\hat{C}_{k}^{(1)} & \cdots & \hat{C}_{k}^{(P)}
\end{array}\right]^{\top} .
$$

Let $\mathbf{V}_{k}$ and $\mathbf{W}_{k}$ be the $P \times P$ complex-valued positive-definite Hermitian and complex symmetric covariance matrices of the estimator $\hat{\mathbf{c}}_{k}$, defined as the so-called circular and noncircular 2nd order moments, respectively, as follows:

$$
\begin{aligned}
& \mathbf{V}_{k} \triangleq \mathbb{E}\left\{\left(\hat{\mathbf{c}}_{k}-\mathbf{c}_{k}\right)\left(\hat{\mathbf{c}}_{k}-\mathbf{c}_{k}\right)^{\mathrm{H}}\right\} \\
& \mathbf{W}_{k} \triangleq \mathbb{E}\left\{\left(\hat{\mathbf{c}}_{k}-\mathbf{c}_{k}\right)\left(\hat{\mathbf{c}}_{k}-\mathbf{c}_{k}\right)^{\top}\right\} .
\end{aligned}
$$

Exact expressions for computing $\mathbf{V}_{k}$ and $\mathbf{W}_{k}$ in the case of input signals with discrete probability distributions and spatially uncorrelated Gaussian noise have been given in 41]. Other expressions for the computation of these covariance matrices are available in the literature for symmetrically distributed sources and also in the general case (c.f. [2] for the former and [14 for the latter). These expressions are very important for the theoretical analysis of the estimator when the model parameters are assumed known, but they have also found application in the context of Gaussianity tests [15]. On the other hand, they can be useful for algorithmic purposes when only output measurements are available. In this case, moments and cumulants can be estimated from time averages, assuming ergodicity.

Let us now define the following real-valued vector:

$$
\mathbf{z}_{k}=\left[\operatorname{Re}\left(\mathbf{c}_{k}\right)^{\top} \quad \operatorname{Im}\left(\mathbf{c}_{k}\right)^{\top}\right]^{\top} \in \mathbb{R}^{2 P \times 1}
$$

where the operators $\operatorname{Re}(\cdot)$ and $\operatorname{Im}(\cdot)$ return the real and imaginary parts of the vector argument, respectively. Consider the estimator $\hat{\mathbf{z}}_{k}$ with covariance matrix $\boldsymbol{\Sigma}_{k} \in \mathbb{R}^{2 P \times 2 P}$, defined as:

$$
\boldsymbol{\Sigma}_{k} \triangleq \mathbb{E}\left\{\left(\hat{\mathbf{z}}_{k}-\mathbf{z}_{k}\right)\left(\hat{\mathbf{z}}_{k}-\mathbf{z}_{k}\right)^{\top}\right\},
$$

which can be readily deduced from (11) and (12), as follows:

$$
\boldsymbol{\Sigma}_{k}=\frac{1}{2}\left(\begin{array}{cc}
\operatorname{Re}\left(\mathbf{V}_{k}+\mathbf{W}_{k}\right) & \operatorname{Im}\left(\mathbf{V}_{k}+\mathbf{W}_{k}\right)^{\top} \\
\operatorname{Im}\left(\mathbf{V}_{k}+\mathbf{W}_{k}\right) & \operatorname{Re}\left(\mathbf{V}_{k}-\mathbf{W}_{k}\right)
\end{array}\right)
$$

We can now define the following scalar multivariate function:

$$
\rho_{k}=\left(\hat{\mathbf{z}}_{k}-\mathbf{z}_{k}\right)^{\top} \boldsymbol{\Sigma}_{k}^{-1}\left(\hat{\mathbf{z}}_{k}-\mathbf{z}_{k}\right)
$$

which depends, though omitted here, on the channel coefficient vector $\mathbf{h}=[h(0) \ldots h(L)]^{\top}$. Porat and Friedlander have been the first to use the above function in the context of channel parameter estimation [39. Actually, it has been shown that if the estimated parameter vector $\hat{\mathbf{h}}$ corresponds to a global minimum of $\rho_{k}$, then $\hat{\mathbf{h}}$ asymptotically tends to the minimum variance estimate [40]. This approach requires the calculation of the covariance matrix $\boldsymbol{\Sigma}_{k}$, which depends on $\mathbf{h}$ and involves knowledge of 
exact output cumulants of order up to eight. Due to the huge computational complexity involved in calculating $\boldsymbol{\Sigma}_{k}$, a simpler solution has been proposed in 22 based on an estimated covariance matrix.

Finally, note that $\mathbf{z}_{k}$ may have some zero elements corresponding to the entries $\left[\mathbf{z}_{k}\right]_{P+p}$ for which $p$ and $k$ are such that the conditions stated in (9) are satisfied. Each zero element in $\mathbf{z}_{k}$ induces a zero row and a zero column in $\boldsymbol{\Sigma}_{k}$. To avoid singularity of the covariance matrix, we need to eliminate the element in position $P+p$ of the vector $\mathbf{z}_{k}$ for each triplet $\left(i_{p}, j_{p}, k\right)$ satisfying (9). This yields a reduced vector $\mathbf{z}_{k} \in \mathbb{R}^{2 P-m_{k}}$, where $m_{k}$ is the number of purely real-valued elements in $\mathbf{c}_{k}$, which gives a $\left(2 P-m_{k}\right) \times\left(2 P-m_{k}\right)$ covariance matrix. This is an important step in order to ensure the nonsingularity of $\boldsymbol{\Sigma}_{k}$. Throughout the rest of this paper, we denote by $\mathbf{z}_{k}$ and $\boldsymbol{\Sigma}_{k}$ the reduced versions of these variables, thus assuming that the covariance matrix is nonsingular.

\subsection{A Chi-square statistic for channel order detection}

Given a sample output data sequence $y(n), n=0, \ldots, N-1$, it can be shown that, as $N$ goes to infinity, the estimator $\hat{\mathbf{c}}_{k}$ tends to a complex multivariate random variable that follows an approximate Gaussian distribution with mean $\mathbf{c}_{k}$ 迥. As a consequence, we have:

$$
\hat{\mathbf{z}}_{k} \sim \mathcal{N}\left(\mathbf{z}_{k}, \boldsymbol{\Sigma}_{k}\right), \quad \text { as } N \rightarrow \infty .
$$

Hence, $\hat{\mathbf{z}}_{k}$ can be viewed as a realization of an asymptotically Gaussian random vector, which can be standardized as follows:

$$
\boldsymbol{\omega}_{k}=\boldsymbol{\Sigma}_{k}^{-1 / 2}\left(\hat{\mathbf{z}}_{k}-\mathbf{z}_{k}\right)
$$

so that $\boldsymbol{\omega}_{k} \in \mathbb{R}^{2 P-m_{k}}$ is asymptotically normal with zero mean and an identity covariance matrix, i.e. $\boldsymbol{\omega}_{k} \sim \mathcal{N}(\mathbf{0}, \mathbf{I})$. The scalar random variable $\rho_{k}$ defined in (16), can now be rewritten as:

$$
\rho_{k}=\boldsymbol{\omega}_{k}^{\top} \boldsymbol{\omega}_{k}
$$

The above results enable us to conclude that $\rho_{k}$ asymptotically follows a Chi-square distribution with $d_{k}=2 P-m_{k}$ degrees of freedom [37, i.e.

$$
\rho_{k} \sim \mathcal{X}_{\left(d_{k}\right)}^{2} .
$$

Therefore, its probability density function (pdf) is given by:

$$
f_{\rho}\left(\rho_{k}\right)= \begin{cases}\frac{1}{2^{d_{k} / 2} \Gamma\left(d_{k} / 2\right)} \rho_{k}^{\left(d_{k} / 2\right)-1} e^{-\rho_{k} / 2}, & \text { for } \rho_{k}>0 \\ 0 & \text { for } \rho_{k} \leq 0,\end{cases}
$$

where $\Gamma(\cdot)$ denotes the well-known Gamma function, defined as

$$
\Gamma(z)=\int_{0}^{\infty} t^{z-1} e^{-t} \mathrm{~d} t
$$

This yields:

$$
\mu_{\rho_{k}}=\mathbb{E}\left\{\rho_{k}\right\}=d_{k}
$$

and

$$
\sigma_{\rho_{k}}^{2}=\mathbb{E}\left\{\left(\rho_{k}-\mu_{\rho_{k}}\right)^{2}\right\}=2 d_{k} .
$$




\subsection{Order detection algorithm}

Contrary to the approach based on an exhaustive search for the minimum variance, our proposition exploits the fact that $\mathbf{z}_{k}=\mathbf{0}$ if $k>L+1$. Thus, replacing the covariance matrix $\boldsymbol{\Sigma}_{k}$ by its estimate, equation (18) becomes:

$$
\hat{\boldsymbol{\omega}}_{k}=\hat{\mathbf{\Sigma}}_{k}^{-1 / 2} \hat{\mathbf{z}}_{k}, \quad \text { for } k>L+1
$$

and we define

$$
\hat{\rho}_{k}=\hat{\boldsymbol{\omega}}_{k}^{\top} \hat{\boldsymbol{\omega}}_{k}=\hat{\mathbf{z}}_{k}^{\top} \hat{\boldsymbol{\Sigma}}_{k}^{-1} \hat{\mathbf{z}}_{k}, \quad k>L+1 .
$$

The above defined variable can be viewed as a measure of the energy in the space of representation of the 4 th-order cumulants. It means that, since $\hat{\mathbf{z}}_{k}$ is a consistent estimator with asymptotically zero bias, $\hat{\rho}_{k}$ can be used to detect the presence of source signals with nonzero 4th-order cumulants when $k \leq L+1$.

In the sequel, we formulate the problem of detecting the channel order as a series of successive hypothesis tests on the estimated variables $\hat{\rho}_{k}, k \in[1, K], K>L$, aiming to determine whether $k>L+1$ or not. From definition (26), we conclude that, as the output data sequence length goes to infinity, $\hat{\rho}_{k}$ tends to be $\mathcal{X}_{\left(d_{k}\right)}^{2}$, if $k>L+1$. In this case, the pdf of $\hat{\rho}_{k}$ is given by (21). However, for $k \leq L+1$, the true cumulant vector $\mathbf{z}_{k}$ is unknown and we cannot center the random vector $\boldsymbol{\omega}_{k}$, hence $\hat{\rho}_{k}$ has a non-central Chi-square distribution, denoted by ${ }_{n C} \chi_{\left(d_{k}\right)}^{2}\left(\lambda_{k}\right)$, with $d_{k}$ degrees of freedom and $\lambda_{k}=\mathbf{z}_{k}^{\top} \boldsymbol{\Sigma}_{k}^{-1} \mathbf{z}_{k}$, related to the mean of the test variable as follows:

$$
\mathbb{E}\left\{\hat{\rho}_{k}\right\}=\lambda_{k}+d_{k} .
$$

From (26), we have:

$$
\hat{\rho}_{k} \sim{ }_{n C} \chi_{\left(d_{k}\right)}^{2}\left(\lambda_{k}\right), \quad \text { with } \begin{cases}\lambda_{k}=0, & k>L+1 \\ \lambda_{k} \neq 0, & k \leq L+1\end{cases}
$$

\section{Remark on the number $d_{k}$ of degrees of freedom}

Situations may arise where the estimated covariance matrix $\hat{\boldsymbol{\Sigma}}_{k}$ is ill-conditioned due, for instance, to negligible values of the cross-correlation between the real and imaginary parts of some of the 4th-order cumulants composing the test statistic $\hat{\rho}_{k}$. Such situations are difficult to predict and the literature lacks guidelines on how to proceed in order to skip them. In spite of that, numerical instabilities can be avoided by controlling the condition number of $\hat{\boldsymbol{\Sigma}}_{k}$, i.e. the ratio between its largest and smallest eigenvalues. In practice, when the condition number is high, we discard the smallest eigenvalues and the associated eigenvectors, until the condition number becomes smaller than a certain threshold. Thus, we get the following reduced eigenvalue decomposition $(\mathrm{EVD})$ of $\hat{\boldsymbol{\Sigma}}_{k} \in \mathbb{R}^{\left(2 P-m_{k}\right) \times\left(2 P-m_{k}\right)}$ :

$$
\hat{\mathbf{\Sigma}}_{k}=\mathbf{U}_{k} \mathbf{D}_{k} \mathbf{U}_{k}^{\top}
$$

where $\mathbf{D}_{k}=\operatorname{Diag}\left(\delta_{1}, \ldots, \delta_{\left.2 P-m_{k}-\mu_{k}\right)}\right.$ and $\mathbf{U}_{k} \in \mathbb{R}^{\left(2 P-m_{k}\right) \times\left(2 P-m_{k}-\mu_{k}\right)}$ is the matrix formed with the eigenvectors of $\hat{\boldsymbol{\Sigma}}_{k}$ associated with its $2 P-m_{k}-\mu_{k}$ largest eigenvalues, $\mu_{k}$ corresponding to the number of smallest eigenvalues discarded in order to attain a moderate condition number. Therefore, the number $d_{k}$ of degrees of freedom of the test statistic $\hat{\rho}_{k}=\hat{\boldsymbol{\omega}}_{k}^{\top} \hat{\boldsymbol{\omega}}_{k}$ is reduced by $\mu_{k}$, i.e.

$$
d_{k}=2 P-m k-\mu_{k} .
$$

In order to show this, note from (29) that we have:

$$
\hat{\boldsymbol{\Sigma}}_{k}^{-1 / 2}=\mathbf{D}_{k}^{-1 / 2} \mathbf{U}_{k}^{\top} \in \mathbb{R}^{d_{k} \times\left(2 P-m_{k}\right)} .
$$


Replacing the above equation in (25), we get:

$$
\hat{\boldsymbol{\omega}}_{k}=\mathbf{D}_{k}^{-1 / 2} \mathbf{U}_{k}^{\top} \hat{\mathbf{z}}_{k} \quad \in \mathbb{R}^{d_{k} \times 1},
$$

and (30) follows from (26). The definition (30) will be used throughout the rest of this paper.

\subsubsection{Hypothesis test and performance analysis}

Let us build our channel order test by defining the null hypothesis $H_{0}(k)$ and the alternative hypothesis $H_{1}(k)$ as follows:

$$
\begin{array}{ll}
H_{0}(k): & k>L+1 \quad \Rightarrow \quad \hat{\rho}_{k} \sim \mathcal{X}_{\left(d_{k}\right)}^{2} \\
H_{1}(k): & k=L+1 \quad \Rightarrow \quad \hat{\rho}_{k} \sim_{n C} \chi_{\left(d_{k}\right)}^{2}\left(\lambda_{k}\right)
\end{array}
$$

Under $H_{0}(k)$, we have $\mathbb{E}\left\{\hat{\rho}_{k}\right\}=d_{k}$ and hence we should expect that $\hat{\rho}_{k}<\eta_{k}$, where $\eta_{k}$ is a decision threshold associated with the number $d_{k}$ of degrees of freedom of the test statistic $\hat{\rho}_{k}$. Under $H_{1}(k)$, $\mathbb{E}\left\{\hat{\rho}_{k}\right\}=\lambda_{k}+d_{k}$, and we should get $\hat{\rho}_{k} \geq \eta_{k}$. The test is successively performed for $k=K, K-1, \ldots, 1$. Our goal is to find the largest value of $k$ so that the null hypothesis $H_{0}(k)$ is rejected, i.e. $\hat{\rho}_{k} \geq \eta_{k}$, which implies $\hat{L}=k-1$. The non-rejection of $H_{0}(k)$ for a given $k$ induces a new test on $\hat{\rho}_{k-1}$. If the null hypothesis is rejected only when $k=1$, then $\hat{L}=0$ and the channel is said to be memoryless. The rejection of $H_{0}(k)$ for all $k \in[1, K]$ indicates that no source signal is present (only Gaussian noise is observed at the antenna output).

Let us denote by $p_{k}$ and $q_{k}$ the probabilities of the event $\hat{\rho}_{k} \geq \eta_{k}$ under hypotheses $H_{0}(k)$ and $H_{1}(k)$, respectively. In other words, for each $k \in[1, K], p_{k}$ and $q_{k}$ are the probabilities of false alarme and detection of the associated test, and are given by:

$$
\begin{array}{ll}
p_{k} \triangleq \mathrm{P}\left[\hat{\rho}_{k} \geq \eta_{k} \mid H_{0}(k)\right], & k \in[1, K], \\
q_{k} \triangleq \mathrm{P}\left[\hat{\rho}_{k} \geq \eta_{k} \mid H_{1}(k)\right], & k \in[1, K] .
\end{array}
$$

In addition, we denote by $P(k)$ the probability of getting $\hat{L}=k-1, k \in[1, K]$, which is defined as the joint probability of the events $\hat{\rho}_{K}<\eta_{K}, \hat{\rho}_{K-1}<\eta_{K-1}, \ldots, \hat{\rho}_{k+1}<\eta_{k+1}$ and $\hat{\rho}_{k} \geq \eta_{k}$, i.e.

$$
P(k)= \begin{cases}\mathrm{P}\left[\hat{\rho}_{K} \geq \eta_{K}\right] ; & \text { for } k=K, \\ \mathrm{P}\left[\hat{\rho}_{K}<\eta_{K} ; \hat{\rho}_{K-1}<\eta_{K-1} ; \ldots ; \hat{\rho}_{k+1}<\eta_{k+1} ; \hat{\rho}_{k} \geq \eta_{k}\right] ; & \text { for } 1 \leq k<K .\end{cases}
$$

For each $k \in[1, K]$, we have one of the two following situations:

i. $\hat{\rho}_{k}<\eta_{k} \Rightarrow \hat{L}<k-1$. The test continues. This happens with probability $1-p_{k}$ (under hypothesis $\left.H_{0}\right)$. The next step is to test $\hat{\rho}_{k-1}$. In this case, if $L \geq k-1$, we underestimate the channel order (missed detection) with unknown probability $1-q_{k}$.

ii. $\hat{\rho}_{k} \geq \eta_{k} \Rightarrow \hat{L}=k-1$. The test stops. This happens with probability $q_{k}$ if $L=k-1$. In this case, the channel order is correctly detected. Otherwise, if $L<k-1$, we overestimate the channel order with probability $p_{k}$.

Theorem 1 The total probability of false alarm (overestimation of the channel order) is given by:

$$
P_{F}=p_{K}+\sum_{k=L+2}^{K-1}\left(1-p_{K}\right) \ldots\left(1-p_{k+1}\right) p_{k}
$$


Proof: Overestimation happens when we get $\hat{L}=k-1$ for any $k>L+1$. Hence, the total probability of channel order overestimation is given by:

$$
P_{F}=\sum_{k=L+2}^{K} \mathrm{P}[\hat{L}=k-1]=\sum_{q=1}^{K-L-1} P(K+1-q) .
$$

Assuming that the events $\hat{\rho}_{k}>\eta_{k}$, for all $k \in[1, K]$, are mutually independent, the joint probability given in (35) can be written as a product of the marginal probabilities of the involved events. Hence, (35) yields:

$$
P(k)= \begin{cases}p_{K}, & k=K, \\ \left(1-p_{K}\right) \ldots\left(1-p_{k+1}\right) p_{k}, & k=K-1, \ldots, L+2\end{cases}
$$

Replacing (38) into (37), equation (36) follows straightforwardly.

Theorem 2 The probability of detection of the channel order is given by:

$$
P_{D}=\left(1-p_{K}\right)\left(1-p_{K-1}\right) \ldots\left(1-p_{L+2}\right) q_{L+1}
$$

Proof: In order to correctly detect the channel order, we need:

$$
\begin{array}{ll}
\hat{\rho}_{K}<\eta_{K}, \hat{\rho}_{K-1}<\eta_{K-1}, \ldots \text { and } \hat{\rho}_{L+2}<\eta_{L+2} & \text { under } H_{0}(k) \text { and } \\
\hat{\rho}_{L+1} \geq \eta_{L+1} & \text { under } H_{1}(k) .
\end{array}
$$

The marginal probability of each of these events can be deduced from (33) for $k=L+2, \ldots, K-1, K$ and from (34) for $k=L+1$. The joint probability of these events is the product of their marginal probabilities, which yields (39).

Theorem 3 The probability of missing the channel order detection (underestimation) is given as follows:

$$
P_{M}=\left(1-p_{K}\right)\left(1-p_{K-1}\right) \ldots\left(1-p_{L+2}\right)\left(1-q_{L+1}\right)
$$

Proof: If the hypothesis $H_{0}(k)$ is not rejected for $k=L+1$, then the channel order is underestimated. This means that $P_{M}=\mathrm{P}\left[\hat{\rho}_{K}<\eta_{K} ; \hat{\rho}_{K-1}<\eta_{K-1} ; \ldots ; \hat{\rho}_{L+2}<\eta_{L+2} ; \hat{\rho}_{L+1}<\eta_{L+1}\right]$, which yields (40).

The total probability of error in the order detection is given by $P_{E}=P_{F}+P_{M}$.

\subsubsection{Decision threshold}

According to the Neyman-Pearson criterion [47, 38], a decision rule can be established in order to maximize the probability of detection $P_{D}$ while not allowing the probability of false alarm $P_{F}$ to exceed a certain value. However, in our case, $P_{F}$ and $P_{D}$ can not be computed explicitly as in (36) and (39), respectively, because we do not know the channel order $L$. We can nevertheless limit the tolerance of each $\hat{\rho}_{k}$-test, by establishing a bound $\alpha$ for an acceptable level of the probability $p_{k}$, defined in (33), so that a decision threshold $\eta_{k}$ can be established in order to ensure that $\mathrm{P}\left[\hat{\rho}_{k} \geq \eta_{k}\right] \leq \alpha, k \in[L+2, K]$.

Considering definition (26), with $d_{k}$ defined in (30), and under the null-hypothesis $(k>L+1)$, the test variable $\hat{\rho}_{k}$ is asymptotically Chi-square distributed with $d_{k}$ degrees of freedom, since $\mathbf{z}_{k}$ is theoretically zero for all $k>L+1$. Otherwise, $\hat{\rho}_{k}$ follows a non-central Chi-square distribution, also with $d_{k}$ degrees of freedom, but with mean equal to $d_{k}+\lambda_{k}$, where $\lambda_{k}=\mathbf{z}_{k}^{\top} \boldsymbol{\Sigma}_{k}^{-1} \mathbf{z}_{k}$ is an unknown parameter, the value 


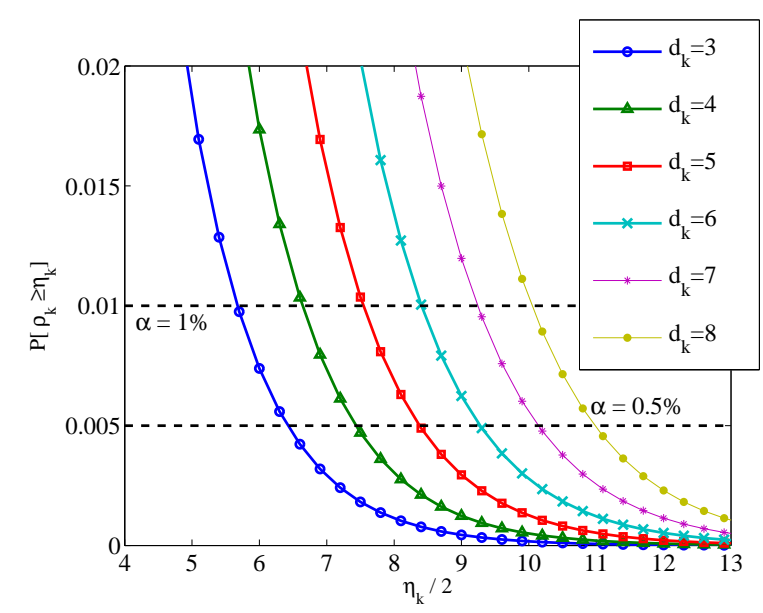

Figure 1: Probability $P\left[\hat{\rho}_{k} \geq \eta_{k}\right]$ as a function of the decision threshold $\eta_{k}$ for different degrees of freedom.

$$
\begin{array}{||c||cc} 
& \alpha=1 \% & \alpha=0.5 \% \\
d_{k}=3 \\
d_{k}=4 \\
d_{k}=5 \\
d_{k}=6 \\
d_{k}=7 \\
d_{k}=8
\end{array}
$$

Table 1: Decision thresholds for two tolerance levels $\alpha$.

of which depends on the true real-valued vector $\mathbf{z}_{k}$. In other words, the distribution of the test variable $\hat{\rho}_{k}$ under the null hypothesis is given by (21), for a given choice of the index set $\mathcal{J}$. Thus, equation (33) becomes:

$$
\begin{aligned}
p_{k} & =\int_{\eta_{k}}^{+\infty} f_{\hat{\rho}}\left(\hat{\rho}_{k}\right) \mathrm{d} \hat{\rho}_{k}, \quad k \in[1, K] \\
& =\frac{1}{2^{d_{k} / 2} \Gamma\left(d_{k} / 2\right)} \int_{\eta_{k}}^{+\infty} \hat{\rho}_{k}^{\left(d_{k} / 2\right)-1} e^{-\hat{\rho}_{k} / 2} \mathrm{~d} \hat{\rho}_{k}
\end{aligned}
$$

Substituting $\hat{\rho}_{k}=2 t$, we have:

$$
\begin{aligned}
p_{k} & =\frac{1}{\Gamma\left(d_{k} / 2\right)} \int_{\eta_{k} / 2}^{+\infty} t^{\left(d_{k} / 2\right)-1} e^{-t} \mathrm{~d} t \\
& =\frac{\bar{\Gamma}\left(d_{k} / 2, \eta_{k} / 2\right)}{\Gamma\left(d_{k} / 2\right)}
\end{aligned}
$$

where $\bar{\Gamma}\left(d_{k} / 2, \eta_{k} / 2\right)$ is the upper incomplete Gamma function 24, defined as

$$
\bar{\Gamma}(z, n)=\int_{n}^{\infty} t^{z-1} e^{-t} \mathrm{~d} t .
$$

Equation (42) enables us to plot the probability curves of the marginal events $\hat{\rho}_{k} \geq \eta_{k}$ for different values of the parameter $d_{k}$, as illustrated in fig. 1.

The decision threshold $\eta_{k}$ can now be established by fixing an acceptable level of tolerance $\alpha$ for the probability of false alarm $p_{k}$ at each $k>L+1$, while allowing $\mathrm{P}\left[\hat{\rho}_{L+1} \geq \eta_{L+1}\right]$ to be as high as possible, depending on the value of $\mathbf{z}_{k}$. In other words, we need $p_{k} \leq \alpha$ for $k>L+1$. Using (42) we conclude that $\eta_{k}$ can be chosen so that

$$
\bar{\Gamma}\left(d_{k} / 2, \eta_{k} / 2\right) \leq \alpha \Gamma\left(d_{k} / 2\right)
$$

In fig. 1, we plot the probability $p_{k}$ as as a function of $\eta_{k} / 2$ using (42), for different values of the number $d_{k}$ of degrees of freedom of the test variable $\hat{\rho}_{k}$. In this example, the tolerance level $\alpha$ has been fixed at two values: $\alpha=1 \%$ and $\alpha=0.5 \%$. The decision threshold can be determined for a given $d_{k}$ by taking the value of $\eta_{k}$ at the point where the corresponding curve crosses a given tolerance level. Values of the decision threshold for the considered values of $d_{k}$ and $\alpha$ are given in Table 1 . 
Table 2: HOS-based channel order detection algorithm

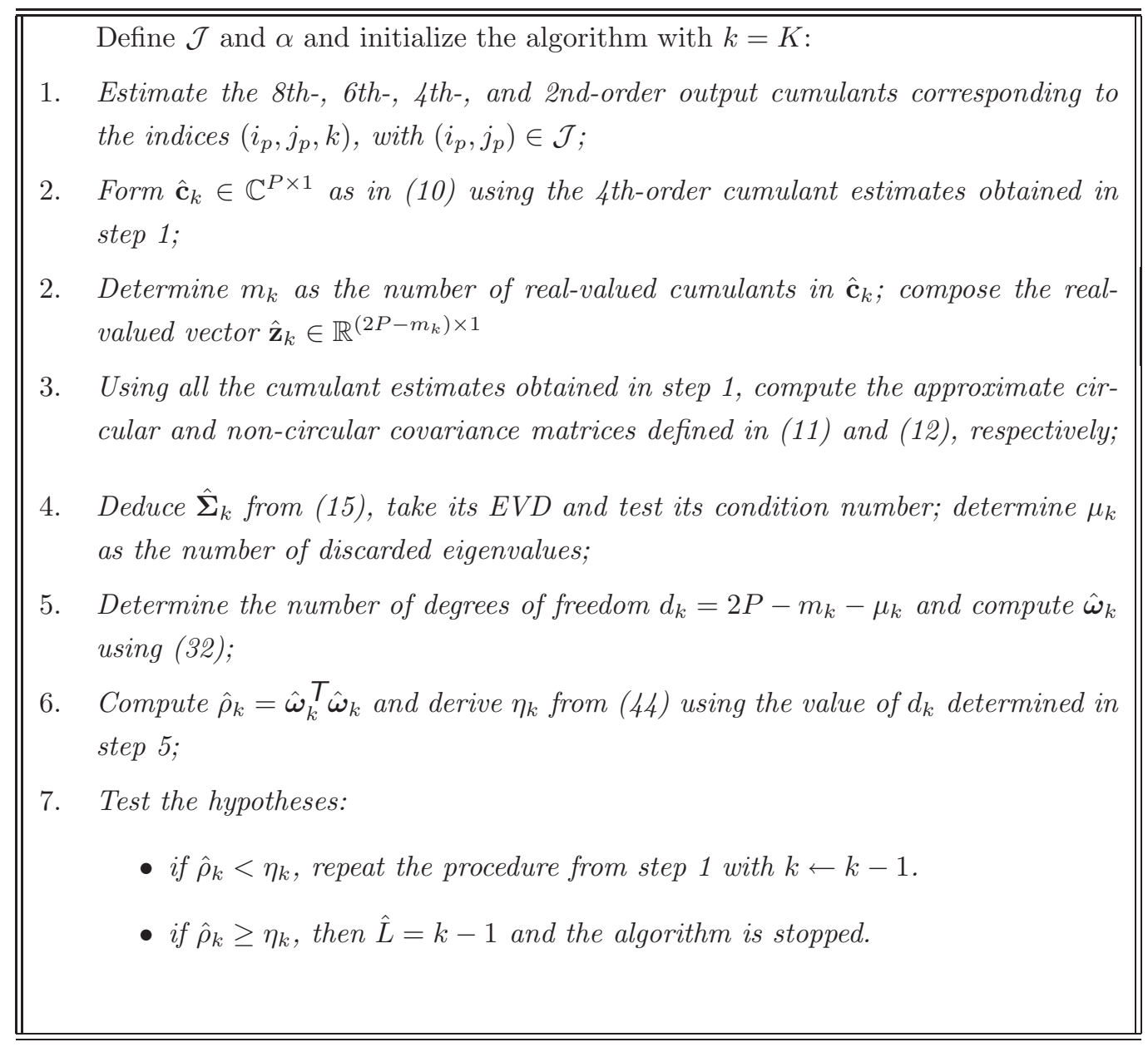

Notice from (37) that the probability of false alarm still depends on $\alpha$ and $K-L$ so that a bad choice of $K$ may reduce the power of the test (increased $P_{F}$ is expected for $K \gg L$ ). Possible solutions to this drawback include the use of the Benjamini-Hochberg procedure for controlling the global level of false alarm (c.f. 10, 11, 91 and references therein). This approach will not be discussed here. The proposed channel order detection algorithm is summarized in Table 2.

\subsubsection{Simulation example}

Let us consider a channel with $L=2$ constant random coefficients drawn from a complex Gaussian distribution. Using $N$ noiseless output data samples, we estimate the 4th-order output cumulants to form the vector $\hat{\mathbf{c}}_{k}$, defined in (10), for $\left(i_{p}, j_{p}\right) \in \mathcal{J}$ and $k=1, \ldots, K$. In this example, we take $\mathcal{J}=\{(1,1) ;(1,2) ; \ldots ;(1, P)\}$ and $P=K=4$. After that, we compose the vector $\hat{\mathbf{z}}_{k}$ by taking the real and imaginary parts of $\hat{\mathbf{c}}_{k}$, as in $(13)$, and then we discard the zero components corresponding to the imaginary part of the purely real-valued cumulants. Any triplet $\left(i_{p}, j_{p}, k\right)$ satisfying the conditions given in (9) yields a purely real-valued cumulant, and we denote by $m_{k}$ the number of such components in the vector $\hat{\mathbf{c}}_{k}$. For $k=4$ and $k=3$, due to our choice of $\mathcal{J}$, we have $m_{4}=m_{3}=1$ (since $C_{3}^{(3)}$ and $C_{4}^{(4)}$ are purely real-valued) and hence $d_{4}=d_{3}=7\left(\mu_{3}=\mu_{4}=0\right.$ in this example).

In order to validate the test variable, we repeated the above described experiment by performing 

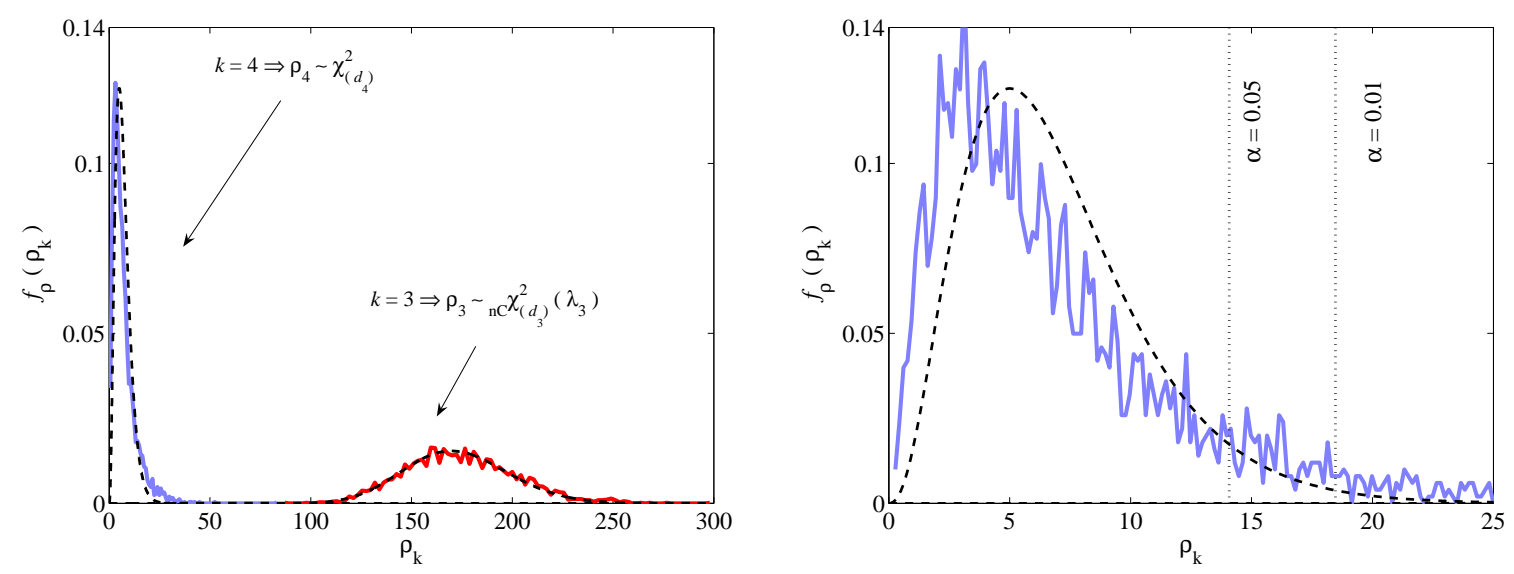

Figure 2: Histogram curves of $\hat{\rho}_{3}$ and $\hat{\rho}_{4}$ (left) in a noiseless SISO channel scenario with $L=2$; detail on the area associated with the null-hypothesis (right). Solid lines correspond to the histogram curves, while dotted lined are the theoretical pdf curves of the associated test variables

3000 Monte Carlo simulations, varying the input data sequence $s(n)$ from one simulation to another, but keeping the same channel parameters over all the simulations (time-invariant channel scenario). In this case, we used $N=10000$ and fig. 目 shows the histogram curves for the test variables $\hat{\rho}_{4}$ and $\hat{\rho}_{3}$ (solid lines). The figure on the right consists in a zoom on the area of the $\hat{\rho}_{4}$ pdf curve on the left. Decision thresholds for $\alpha=1 \%$ and $\alpha=5 \%$ are depicted. The dotted lines correspond to the theoretical pdf curves of the associated variables. The curves for $k<3$ have been omitted from fig. 目 because for $k=1$ and $k=2$ we have $\lambda_{k} \gg \eta_{k}$ (none underestimation cases occurred).

\section{Blind MISO channel Order detection and parameter estima- tion}

In this section, we consider several source signals sharing the same carrier frequency at the neighborhood of the receive antenna. We are interested in estimating the number of sources and the respective channel parameters. In this context, the radiocommunication channel can be modeled as a MISO system. First, we reformulate the cumulant vector definitions for the MISO case, and then we show that the SISO channel detection order method introduced in the last section can be used to determine the order of the longest channel in the MISO model. After that, we introduce two channel parameter estimation algorithms that allow us to reconstruct the output cumulants. Using a deflation-based approach, the procedure iterates by restarting the order detection method on the second longest channel in the MISO model.

We assume that an unknown number $Q$ of co-channel users are located far apart from each other, hence utilizing physically different channels. The signal measured at time instant $n$ at the receive antenna, is written as:

$$
\begin{gathered}
x_{q}(n)=\sum_{\ell=0}^{L_{q}} h_{q}(\ell) s_{q}(n-\ell), \\
y(n)=\sum_{q=1}^{Q} x_{q}(n)+v(n),
\end{gathered}
$$


where

$$
h_{q}(0)=1
$$

and each channel $q \in[1, Q]$ has memory $L_{q}+1$. Assumptions A1 to A4 from section 2 still hold for each source $s_{q}(n)$ and their respective channel coefficients $h_{q}(\ell)$. Here, we further assume that

A5 : The input signals $s_{q}(n)$ are mutually (spatially and temporally) independent with nonzero Kurtoses.

A6 : The channel orders are not equal and all bounded by a known $K$; without loss of generality, we consider that $K>L_{1}>\ldots>L_{Q}$, so that $L_{1}=\max _{1 \leq q \leq Q}\left(L_{q}\right)$.

The case where some channels have the same length will be briefly discussed in section 3.3 .

Taking the above assumptions into account and using the multilinearity property of cumulants, the 4th-order cumulant of the output signal $y(n)$, defined in (3), can be expressed as the sum of the marginal cumulant contributions of each source, as follows:

$$
c_{4, y}(i, j, k)=\sum_{q=1}^{Q} C_{q}(i, j, k)
$$

in which $C_{q}(i, j, k)$ depends on the unknown channel parameters $h_{q}(\ell)$ and can be written as in (田:

$$
C_{q}(i, j, k)=\gamma_{4, s_{q}} \sum_{\ell=0}^{L_{q}} h_{q}^{*}(\ell) h_{q}(\ell+i) h_{q}^{*}(\ell+j) h_{q}(\ell+k), \quad q \in[1, Q],
$$

where $\gamma_{4, s_{q}}=c_{4, s_{q}}(0,0,0)$. Since we assume that $h_{q}(\ell)=0, \forall \ell \notin\left[0, L_{q}\right]$, we have:

$$
C_{q}(i, j, k)=0, \quad \forall|i|,|j|,|k|>L_{q} .
$$

Using (47), we can define the 4th-order output cumulant vector, as follows:

$$
\mathbf{c}_{k} \triangleq \sum_{q=1}^{Q} \mathbf{c}_{k, q} \quad \in \mathbb{C}^{P \times 1}, \quad \text { for each } k \in[1, K],
$$

where

$$
\left[\mathbf{c}_{k, q}\right]_{p} \triangleq C_{q}\left(i_{p}-1, j_{p}-1, k-1\right), \text { with } q \in[1, Q], p \in[1, P] \text {, and }\left(i_{p}, j_{p}\right) \in \mathcal{J},
$$

the index set $\mathcal{J}=\left\{\left(i_{1}, j_{1}\right) ; \ldots ;\left(i_{P}, j_{P}\right)\right\}$ being formed of strictly positive integer numbers $i_{p}, j_{p} \leq K$. Note from (49) that

$$
\mathbf{c}_{k, q}=\mathbf{0}_{P}, \forall k>L_{q}+1 .
$$

Hence, since we have assumed $L_{1}>\ldots>L_{Q}$, we have:

$$
\mathbf{c}_{k}=\left\{\begin{array}{lc}
\mathbf{0}_{P}, & \text { if } k>L_{1}+1 \\
\mathbf{c}_{k, 1}, & \text { if } L_{2}+1<k \leq L_{1}+1 \\
\mathbf{c}_{k, 1}+\mathbf{c}_{k, 2}, & \text { if } L_{3}+1<k \leq L_{2}+1 \\
\vdots & \vdots \\
\mathbf{c}_{k, 1}+\ldots+\mathbf{c}_{k, Q}, & \text { if } k \leq L_{Q}+1 .
\end{array}\right.
$$

In the sequel, we describe a deflation-type approach based on the use of nested channel order detectors, in which the test statistics are formed from the residual 4th-order output cumulants, after subtracting the contribution of the previously estimated channels. We remark that, in the context of $2 \times 1$ MISO systems, a description of this deflation-based technique was proposed in [20], using a different order detection method, with test statistics obtained from the eigenvalue decomposition of a cumulant matrix. 


\subsection{Nested MISO order-detectors and blind channel identification}

Equation (53) shows that, for an appropriate range of $k$, the output cumulant vector contains information on the longest channel exclusively. More precisely, if $k=L_{1}+1, \mathbf{c}_{k}$ can be written only in terms of the coefficients $h_{1}(\ell)$. As a result, if we can determine the order $L_{1}$ of the longest channel, then $\hat{\mathbf{c}}_{L_{1}+1,1}=\hat{\mathbf{c}}_{L_{1}+1}$ and we can estimate the channel coefficients associated with the source $q=1$. After that, using the estimated channel coefficients, the marginal cumulants associated with the detected source can be reconstructed approximately for all $k \in\left[1, L_{1}+1\right]$. To this end, Kurtosis estimation is also needed. Then, by subtracting the contribution $\hat{\mathbf{c}}_{k, 1}$ from $\hat{\mathbf{c}}_{k}$, we get an identical situation with $Q-1$ sources and the same processing can be repeated until the $Q$ channels are identified. The algorithm is stopped when no significant residual information remains in the estimated output cumulant vector.

The above suggested procedure summarizes the idea behind the proposed nested MISO detectors. The three following main steps are repeated for each user $q \in[1, Q]$ :

1. Channel order detection: determine $L_{q}$;

2. Blind channel identification: estimate channel coefficients $\hat{h}_{q}(\ell), \ell \in\left[0, L_{q}\right]$;

3. Estimation of marginal cumulants: reconstruct $\hat{\mathbf{c}}_{k, q}$ for all $k \in\left[1, L_{q}+1\right]$ using the estimated channel coefficients.

Before proceeding to user $q+1$, the marginal contribution of user $q$ is subtracted from the estimated output cumulant vector $\hat{\mathbf{c}}_{k}$. Using the channel-order detection method proposed in section 2, we can solve the problem of determining the channel order $L_{q}$ by formulating a multiple hypothesis test based on the variables $\hat{\rho}_{k}$ that are computed from the estimated MISO channel output cumulant vectors, $\hat{\mathbf{c}}_{k}$. Details about these test variables (step 1) are postponed to section 3.2. In the sequel, we discuss the estimation of the channel coefficients $\hat{h}_{q}(\ell)$ (step 2). Afterwards, we will show that the reconstruction of the marginal cumulant vectors $\hat{\mathbf{c}}_{k, q}$ for all $k \in\left[1, L_{q}+1\right]$ (step 3 ) is straightforward.

\subsubsection{MISO channel parameter estimation}

In this section, we assume that the channel order $L_{q}$ as well as the marginal 4th-order cumulants $C_{q}\left(i_{p}-1, j_{p}-1, L_{q}\right)$ are known, $\left(i_{p}, j_{p}\right) \in \mathcal{J}, q \in[1, Q]$. In the following, we present two methods for estimating the marginal channel parameters $\hat{h}_{q}(\ell)$ associated with the source $q, \ell \in\left[0, L_{q}\right]$.

\section{A. Rank-1 approximation-based method}

From (48), taking $\left(i_{p}, j_{p}\right) \in \mathcal{J}$ and $k=L_{q}$, we get:

$$
C_{q}\left(i_{p}-1, j_{p}-1, L_{q}\right)=\gamma_{4, s_{q}} h_{q}\left(i_{p}-1\right) h_{q}^{*}\left(j_{p}-1\right) h_{q}\left(L_{q}\right), \quad q \in[1, Q],
$$

where we have used the constraint (46). Hence,

$$
\mathbf{c}_{L_{q}+1, q} \triangleq \gamma_{4, s_{q}} h_{q}\left(L_{q}\right) \mathbf{g}^{(q)} \in \mathbb{C}^{P \times 1},
$$

where

$$
\left[\mathbf{g}^{(q)}\right]_{p} \triangleq h_{q}\left(i_{p}-1\right) h_{q}^{*}\left(j_{p}-1\right), \quad p \in[1, P] .
$$

In order to recover the channel parameters, we need to impose some minimal conditions on the index set $\mathcal{J}$ of the cumulants utilized by the algorithm. Simple conditions ensuring correct parameter estimation are as follows:

$$
\begin{cases}i_{p}=p, & \forall p \in[1, P], P=K, \\ j_{p}=1, & \forall p \in[1, P], P=K .\end{cases}
$$


Satisfying the above conditions leads to

$$
\left[\mathbf{g}^{(q)}\right]_{p}=h_{q}(p-1)
$$

and, for $p \in[1, P],(55)$ becomes

$$
\mathbf{c}_{L_{q}+1, q}=\gamma_{4, s_{q}} h_{q}\left(L_{q}\right) \mathbf{h}^{(q)},
$$

with

$$
\mathbf{h}^{(q)}=\left[1, h_{q}(1), \ldots, h_{q}\left(L_{q}\right), 0, \ldots, 0\right]^{\top} \in \mathbb{C}^{K \times 1} .
$$

Thus we can construct the matrix $\mathbf{C}_{q} \in \mathbb{C}^{K \times K}$, as follows:

$$
\mathbf{C}_{q} \triangleq \mathbf{c}_{L_{q}+1, q} \mathbf{c}_{L_{q}+1, q}^{\mathrm{H}}=\gamma_{4, s_{q}}^{2}\left|h_{q}\left(L_{q}\right)\right|^{2} \mathbf{h}^{(q)} \mathbf{h}^{(q)}{ }^{\mathrm{H}},
$$

which is clearly a rank-1 matrix. Assuming that we know the channel order $L_{q}$, we can estimate the vector $\mathbf{h}^{(q)} \in \mathbb{C}^{K \times 1}$, up to a complex scaling factor, by computing the eigenvector associated with the sole nonzero eigenvalue of $\mathbf{C}_{q}$. The constraint $h_{q}(0)=1$ imposed in (46) allows us to avoid the trivial solution and eliminate the intrinsic scaling ambiguity. In practice, we only need to use the first $L_{q}+1$ elements of $\hat{\mathbf{c}}_{L_{q}+1, q}$ to compose $\hat{\mathbf{C}}_{q}$ in order to avoid the zero-padding at the estimated channel tail.

By allowing for an increased set of 4th-order cumulants, we can improve the quality of the channel parameter estimates. It can be particularly interesting to expand the index set $\mathcal{J}$ to the following:

$$
\mathcal{J}=\{(1,1) \ldots(K, 1) \quad \ldots \quad(1, K) \ldots(K, K)\}
$$

so that $P=K^{2}$. Using the above index set, we get the following equation from (55):

$$
\mathbf{B}_{q} \triangleq \operatorname{unvec}\left(\mathbf{c}_{L_{q}+1, q}, K\right)=\gamma_{4, s_{q}} h_{q}\left(L_{q}\right) \mathbf{G}^{(q)},
$$

with $\mathbf{G}^{(q)}=$ unvec $\left(\mathbf{g}^{(q)}, K\right)=\mathbf{h}^{(q)} \mathbf{h}^{(q)}{ }^{\mathrm{H}}$, where the notation unvec $(\mathbf{x}, n)$ stands for the unvectorization operator, which builds a matrix $\mathbf{X} \in \mathbb{C}^{m \times n}$ from the vector argument $\mathbf{x} \in \mathbb{C}^{m n \times 1}$. As a result, we can still use the rank-1 approximation solution to estimate the channel vector $\mathbf{h}^{(q)}$ by taking the singular vector associated with the largest singular value of $\mathbf{B}_{q}$.

\section{B. Optimal solution in the total least squares sense}

Recalling (54), we notice that:

$$
\left[\mathbf{c}_{L_{q}+1, q}\right]_{p}=C_{q}\left(i_{p}-1, j_{p}-1, L_{q}\right) .
$$

Thus, another way to recover the channel coefficients consists of solving the following linear system of equations, obtained from (54):

$$
\begin{gathered}
C_{q}\left(v-1, u-1, L_{q}\right) h_{q}(w-1)-C_{q}\left(w-1, u-1, L_{q}\right) h_{q}(v-1)=0, \\
\text { with } 1 \leq v<w \leq L_{q}+1, \quad \text { and } \quad 1 \leq u \leq L_{q}+1 .
\end{gathered}
$$

From (65) we can build a set of up to $L_{q}\left(L_{q}+1\right)^{2} / 2$ equations with $L_{q}$ unknowns, which can be rewritten in a matrix form as follows:

$$
\mathbf{T}_{q} \mathbf{h}_{q}=\mathbf{0}
$$


where $\mathbf{0}$ is an all-zero vector, $\mathbf{h}_{q}=\left[1, h_{q}(1), \ldots, h_{q}\left(L_{q}\right)\right]^{\top}$ and matrix $\mathbf{T}_{q}$ is composed of the output cumulants with $L_{q}+1$ columns and up to $L_{q}\left(L_{q}+1\right)^{2} / 2$ rows, each row having only two nonzero elements given by

$$
\left\{\begin{array}{l}
{\left[\mathbf{T}_{q}\right]_{r v}=-C_{q}\left(w-1, u-1, L_{q}\right),} \\
{\left[\mathbf{T}_{q}\right]_{r w}=C_{q}\left(v-1, u-1, L_{q}\right),}
\end{array}\right.
$$

where $r=\left(L_{q}+1\right)(v+w-3)+u$ is the row number with $v, w$ and $u$ satisfying (66).

Equation (67) can be solved by computing the right singular vector of $\mathbf{T}_{q}$ associated with its smallest singular value. Forming the index set $\mathcal{J}$ from (57), we have $1 \leq v<w \leq L_{q}+1$ and $u=1$, and we may get up to $L_{q}\left(L_{q}+1\right) / 2$ equations. The expanded index set given in (62) can also be used to improve the estimation quality. This solution has been originally proposed in [13] and is shown to be optimal in the total least squares (TLS) sense.

In terms of computation burden both MISO channel parameter estimation methods presented in this section have the cost of performing one SVD for each channel $q \in[1, Q]$. For comparison purposes, consider in both cases the index set $\mathcal{J}$ formed from (57). The Rank-1 approximation-based method performs the SVD of a Hermitian matrix of dimensions $\left(L_{q}+1\right) \times\left(L_{q}+1\right)$, while the TLS approach computes the SVD of a complex-valued matrix of dimensions $\left(L_{q}\left(L_{q}+1\right) / 2\right) \times\left(L_{q}+1\right)$. Due to the larger number of equations used, the TLS method may theoretically give better results at the cost of an increased complexity (except for the cases $L_{q}=1, L_{q}=2$ ). Results shown in section 4 indicate that, for those particular cases, the performance gap between these two methods may be negligible.

\subsubsection{Composition of marginal cumulants from estimated channel coefficients}

Rewriting (53) for $k=L_{q}+1, q \in[1, Q]$, we have:

$$
\mathbf{c}_{k}=\left\{\begin{array}{lc}
\mathbf{c}_{L_{1}+1,1} & \text { for } k=L_{1}+1 \\
\mathbf{c}_{L_{2}+1,1}+\mathbf{c}_{L_{2}+1,2} & \text { for } k=L_{2}+1 \\
\vdots & \vdots \\
\mathbf{c}_{L_{Q}+1,1}+\ldots+\mathbf{c}_{L_{Q}+1, Q} & \text { for } k=L_{Q}+1
\end{array}\right.
$$

so that we can estimate the marginal cumulant contribution of source $q$ as follows:

$$
\hat{\mathbf{c}}_{L_{q}+1, q}=\hat{\mathbf{c}}_{L_{q}+1}-\sum_{i=1}^{q-1} \overline{\mathbf{c}}_{L_{q}+1, i}
$$

where $\overline{\mathbf{c}}_{L_{q}+1, i}$ are the reconstructed cumulant vectors obtained from (48) using the previously estimated coefficient vectors $\hat{\mathbf{h}}_{i}, i \in[1, q-1]$. To achieve this step, we need to know the Kurtosis of source $q$. Note that $\left[\hat{\mathbf{c}}_{\hat{L}_{q}+1, q}\right]_{p}=\hat{C}_{q}\left(i_{p}-1, j_{p}-1, \hat{L}_{q}\right)$. From (54), using the index set defined in (57) and imposing the constraint (46), we get:

$$
\left[\hat{\mathbf{c}}_{\hat{L}_{q}+1, q}\right]_{p}=\hat{\gamma}_{4, s_{q}} \hat{h}_{q}(p-1) \hat{h}_{q}\left(\hat{L}_{q}\right) .
$$

Thus, an estimate of the source Kurtosis $\gamma_{4, s_{q}}$ is given by the Least Squares solution:

$$
\hat{\gamma}_{4, s_{q}}=\frac{\sum_{p} \hat{h}_{q}^{*}(p-1)\left[\hat{\mathbf{c}}_{\hat{L}_{q}+1, q}\right]_{p}}{\hat{h}_{q}\left(\hat{L}_{q}\right) \sum_{p}\left|\hat{h}_{q}(p-1)\right|^{2}}, \quad q \in[1, Q] .
$$




\subsection{Test statistics for MISO order-detection}

To start with the MISO channel detection procedure proposed above, we must first determine the order of each user channel, since all the subsequent steps depend on this parameter. Here, we treat this problem as an FIR channel order selection problem and show that we can use the method proposed in section 2 by computing the test variables from the estimated cumulants obtained at the output of the MISO channel.

Recalling definition (26), we can compute the test variable $\hat{\rho}_{k}$ from the real-valued vector $\hat{\mathbf{z}}_{k}$, which is defined in (13) and consists of the real and imaginary parts of the vector $\hat{\mathbf{c}}_{k}$ of the estimated 4th-order cumulants of the MISO channel output, defined in (50). Hence, we can write

$$
\hat{\mathbf{z}}_{k} \triangleq \sum_{q=1}^{Q} \hat{\mathbf{z}}_{k, q}
$$

and

$$
\hat{\mathbf{z}}_{k, q} \triangleq\left[\operatorname{Re}\left(\hat{\mathbf{c}}_{k, q}\right)^{\top} \operatorname{Im}\left(\hat{\mathbf{c}}_{k, q}\right)^{\top}\right]^{\top}, \quad q \in[1, Q] .
$$

Thus, from (53), we deduce that

$$
\hat{\mathbf{z}}_{k}=\hat{\mathbf{z}}_{k, 1}, \quad \text { for } k=L_{1}+1 .
$$

In addition, since $\mathbf{z}_{k}=\mathbf{0}_{d_{k}}$ for $k>L_{1}+1$, we can use (26) to construct our test variable from the vector $\hat{\mathbf{z}}_{k}$, so that, for $k>L_{1}+1, \hat{\rho}_{k}$ follows a Chi-square distribution with $d_{k}$ degrees of freedom, with $d_{k}$ given by $(30)$.

On the other hand, as long as $\mathbf{z}_{k}$ is nonzero for $k=L_{1}+1$, the variable $\hat{\rho}_{L_{1}+1}$ has a non-central Chi-square distribution with $\lambda_{L_{1}+1}=\mathbf{z}_{L_{1}+1,1}^{\top} \boldsymbol{\Sigma}_{L_{1}+1}^{-1} \mathbf{z}_{L_{1}+1,1}$, related to the mean of the test variable as follows: $\mathbb{E}\left\{\hat{\rho}_{L_{1}+1}\right\}=\lambda_{L_{1}+1}+d_{L_{1}+1}$. We can hence denote:

$$
\hat{\rho}_{k}=\hat{\mathbf{z}}_{k}^{\top} \hat{\boldsymbol{\Sigma}}_{k}^{-1} \hat{\mathbf{z}}_{k} \begin{cases}\sim \chi_{\left(d_{k}\right)}^{2}, & k>L_{1}+1, N \rightarrow \infty \\ \sim{ }_{n C} \chi_{\left(d_{k}\right)}^{2}\left(\lambda_{k}\right), & k \leq L_{1}+1, N \rightarrow \infty\end{cases}
$$

Using (15), we can deduce $\hat{\boldsymbol{\Sigma}}_{k}$ for $k \geq L_{1}+1$ from the estimated circular and non-circular covariance matrices, given in (11) and (12), respectively.

Equation (76) shows that the test statistic $\hat{\rho}_{k}$ enables us to correctly detect the order of the longest channel associated with the MISO mixture. After determining the order $L_{1}$, we can use one of the techniques described in section 3.1 to estimate the channel coefficients $\hat{h}_{1}(\ell)$. Then, by replacing the estimated coefficients in (48), we reconstruct the vectors $\overline{\mathbf{c}}_{k, 1}$ for all $k \in\left[1, L_{1}+1\right]$. Subtracting $\overline{\mathbf{c}}_{k, 1}$ from $\hat{\mathbf{c}}_{k}$ allows for the computation of new test statistics leading to the detection of the next longest channel. This deflation principle, discussed in section 3.1, allows us to successively extract the sources from the MISO mixture. The algorithm continues until no significant residual information is detected in the remaining cumulant vector. The nested MISO channel detection method is summarized in Table 3.

\subsection{MISO channels with identical lengths}

In the case where at least two channels, $q_{1}$ and $q_{2}$, have the same order, $L_{q_{1}}=L_{q_{2}}=L$, the proposed MISO channel detection method does not fully apply because the residual cumulant $\hat{\mathbf{c}}_{k}$ for $k=L+1$ contains information from both channels, i.e.

$$
\hat{\mathbf{c}}_{L+1}=\hat{\mathbf{c}}_{L+1, q_{1}}+\hat{\mathbf{c}}_{L+1, q_{2}} \text {. }
$$

As long as the marginal cumulants are not separable, the deflation technique is no longer suitable to estimate the channel parameters. Hence, the hypothesis test for $q_{1}$ should lead to the same result as 
Table 3: Nested MISO channel detection procedure

1. Estimate the 4th-order MISO channel output cumulants; compose the real-valued vectors $\hat{\mathbf{z}}_{k}$, and compute $\hat{\Sigma}_{k}, k \in[1, K]$;

2. Initialize $q=1$ and $k=K$;

3. Compute the test variables $\hat{\rho}_{k}$, defined in (26) using $\hat{\mathbf{z}}_{k}$ and $\hat{\boldsymbol{\Sigma}}_{k}$; determine the decision threshold using the procedure described in section

4. Run the order-detection algorithm by testing the hypotheses:

if $\hat{\rho}_{k}<\eta_{k}$ then take $k \leftarrow k-1$ and start over from step 3 .

if $\hat{\rho}_{k} \geq \eta_{k}$ then $\hat{L}_{q}=k-1$. Go to step 5 .

if $\hat{L}_{q}=0$, stop the procedure;

5. Compute $\hat{h}_{q}(\ell), \ell \in\left[0, L_{q}\right]$, from $\hat{\mathbf{c}}_{L_{q}+1, q}$ using one of two the blind channel identification techniques described in section 3.1.

6. From (48), compute the entries of $\overline{\mathbf{c}}_{k, q}$, for all $k \in\left[1, L_{q}+1\right]$, using $\hat{h}_{q}(\ell)$ and $\hat{\gamma}_{4, s_{q}}$, the latter being obtained from $(72)$;

7. Update the output cumulant vector as $\hat{\mathbf{c}}_{k} \leftarrow \hat{\mathbf{c}}_{k}-\overline{\mathbf{c}}_{k, q}$;

8. Update $q \leftarrow q+1$ and repeat steps 3 to 8 until hypothesis $\hat{\rho}_{k}<\eta_{k}$ is not rejected in step 4 .

that of $q_{2}$, i.e. $\hat{L}_{q_{1}}=\hat{L}_{q_{2}}$. In this case, the estimated parameters should be disregarded and the nested detection procedure terminated. Therefore, the proposed approach can detect the order and estimate the parameters of the channels having order higher than $L+1$. The procedure is not able to identify the number of channels with the same length $L+1$, but can indicate that at least two sources are present in the MISO mixture.

Nevertheless, cumulants are also sensitive to the nonlinearity of a stochastic process, in the sense defined in [23]. Yet, the process obtained by MISO linear filtering of several i.i.d. sequences cannot be obtained by linear filtering of a single i.i.d. sequence. This property will be used in a future paper to detect the number of sources when channels may have the same length.

\section{Simulation results}

In this section, we illustrate the performance of the nested detectors described in Table 3 by means of some simulation results. In order to evaluate the proposed detection/identification method, we make use of the two following criteria, which are defined for each signal source $q \in[1, Q]$ :

$i$. The empirical probability of detection: it corresponds to the ratio between the number of Monte Carlo simulations in which the detector results are consistent $\left(\hat{L}_{q}=L_{q}\right)$ and the total number $R$ of realizations of the experiment;

ii The normalized mean squared error (NMSE) of the consistent results: this metric is computed for each channel $q$ as follows:

$$
\operatorname{NMSE}(q)=\frac{1}{R_{q}} \sum_{r=1}^{R_{q}} \frac{\left\|\widehat{\mathbf{h}}_{q}^{\langle r\rangle}-\mathbf{h}_{q}\right\|^{2}}{\left\|\mathbf{h}_{q}\right\|^{2}},
$$



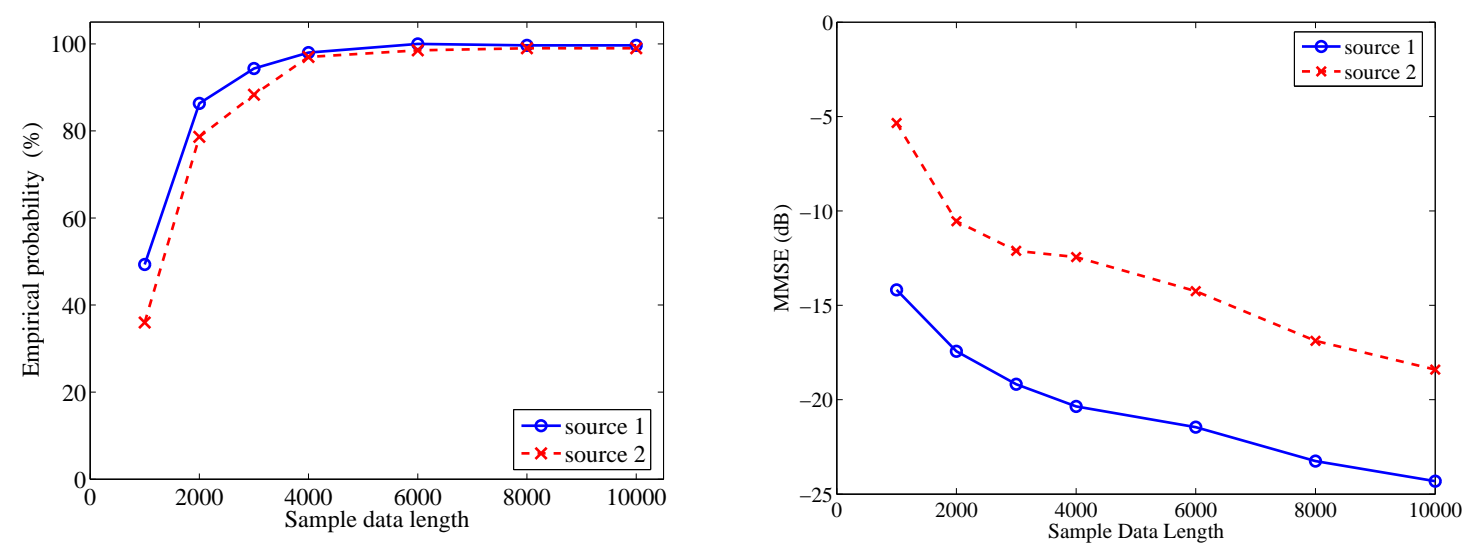

Figure 3: Empirical probability of detection (left) and NMSE (right) versus the sample data length, with $\mathrm{SNR}=40 \mathrm{~dB}\left(L_{1}=2, L_{2}=1\right.$ and $\left.K=5\right)$.

where $R_{q}$ is the number of consistent results obtained for source $q$ over $R$ realizations and $\widehat{\mathbf{h}}_{q}^{\langle r\rangle}$ is the estimated channel vector associated with source $q$, obtained for the (consistent) realization $r$.

Both blind channel identification algorithms described in section 3.1 have been implemented (rank-1 approximation and TLS) using the index set defined in (62), assuming the input signal constellation is known (none Kurtosis estimation). However, since the results are very close, we omitted here the curves obtained with the rank-1 approximation solution.

\section{Nested algorithm for blind order detection and parameter estimation of MISO channels}

Let us consider a frequency-selective MISO communication channel with $Q=2$ users and one single receive antenna. A static multipath propagation scenario is assumed, which induces a delay spread of the order of 3 symbol periods on the channel of source $1\left(L_{1}=2\right)$ and of the order of 2 symbol periods on the channel of source $2\left(L_{2}=1\right)$. Channel coefficients have been randomly generated with a continuous complex Gaussian distribution. The figures in the sequel have been obtained with the following coefficient vectors:

$$
\begin{aligned}
& \mathbf{h}_{1}=\left[\begin{array}{lll}
1.0, & 1.35-0.57 \jmath, & -0.72+1.49 \jmath
\end{array}\right]^{\top}, \\
& \mathbf{h}_{2}=\left[\begin{array}{ll}
1.0, & -1.14+0.23 \jmath
\end{array}\right]^{\top} .
\end{aligned}
$$

A known upper bound of $K=5$ is assumed for both channel orders. The source signals have been QPSK modulated and 200 input data blocks have been independently generated, with $N$ samples per block. The results shown below have been averaged over the simulations for all received data blocks, using the nested detection-estimation procedure presented in Table 3.

First, we fixed the SNR value at $40 \mathrm{~dB}$ and considered an output sample data length $(N)$ varying from $10^{3}$ to $10^{4}$. At the left-hand side of fig. 3 , we notice a poor detection performance for both sources when the sample data length is smaller than $N=2000$. The overall channel identification performance is improved as the number of output data samples increases. This is an expected result due to the fact that the cumulant estimators are biased, but the bias is asymptotically zero. Increased cumulant estimation variance implies worse channel parameter estimation, specially for short data lengths. In addition, in fig. 3, we observe a performance loss between sources 1 and 2, suggesting that the proposed algorithm 

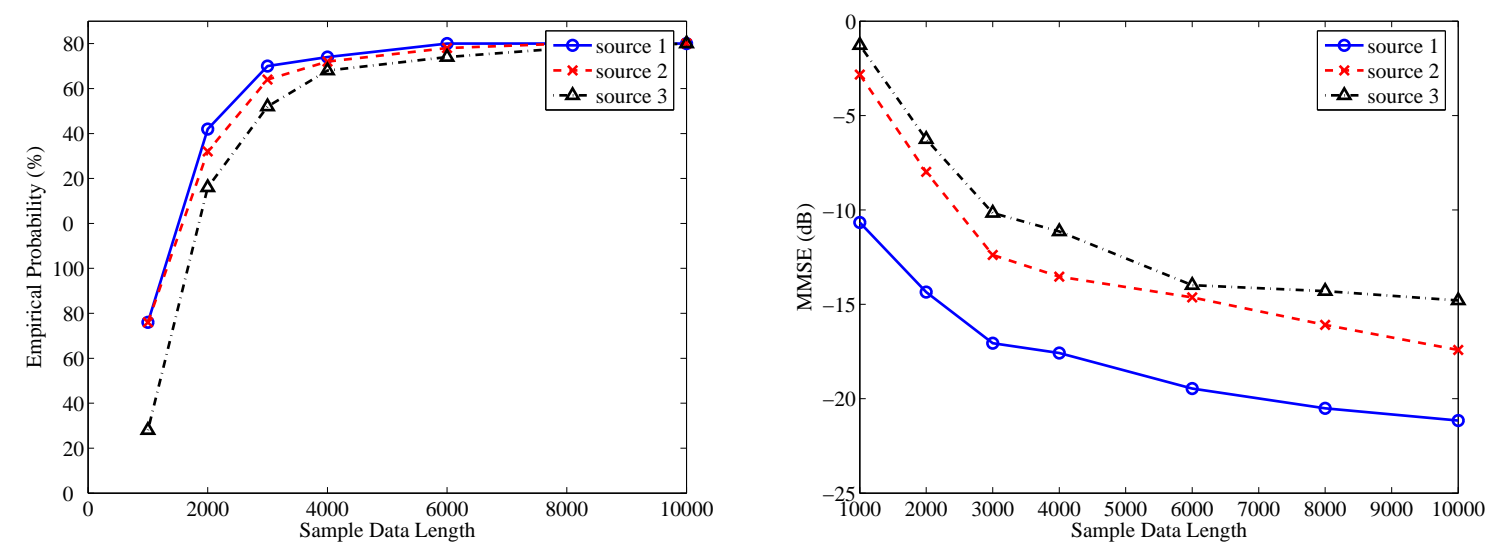

Figure 4: Empirical probability of detection (left) and NMSE (right) versus the sample data length, with $\mathrm{SNR}=40 \mathrm{~dB}\left(L_{1}=3, L_{2}=2, L_{3}=1\right.$ and $\left.K=5\right)$.

suffers from error propagation, harming the identification of shorter channels.

Afterwards, we considered a channel scenario with $Q=3$ sources, where $L_{1}=3, L_{2}=2$ and $L_{3}=1$. Representing the randomly generated channel coefficient vectors by $\mathbf{g}_{1}, \mathbf{g}_{2}$ and $\mathbf{g}_{3}$, we used:

$$
\mathbf{g}_{1}=\left[\begin{array}{ll}
1.0,-0.5783+0.7291 \jmath, & 1.3820-0.99 \jmath,-0.6140+1.3799 \jmath
\end{array}\right]^{\top},
$$

$\mathbf{g}_{2}=\mathbf{h}_{1}$, and $\mathbf{g}_{3}=\mathbf{h}_{2}$. Again, the $\mathrm{SNR}$ value was fixed at $40 \mathrm{~dB}$ and the output sample data length $(N)$ varied from $10^{3}$ to $10^{4}$. Fig. $⿴$ (left) shows the empirical probability of detection for each source, highlighting the fact that a too small data length entails performance degradations. We also verified an improvement of channel identification performance as the sample data length increases, as it can be seen at the right-hand side of fig. 4 . Here again, we can observe the effects of error propagation on the estimation of the shorter channel parameters. This behavior can be explained from the fact that shorter channels are estimated from the residual 4th-order cumulants, i.e. the information remaining after the subtraction of the reconstructed cumulants of longer channels, which are themselves estimated and hence susceptible to errors.

In another simulation, we used channels $\mathbf{h}_{1}$ and $\mathbf{h}_{2}$ with a sample data length of $N=10^{4}$ for SNR values ranging from 5 to $40 \mathrm{~dB}$. As we can see in fig. 5 , the nested detectors are quite robust with respect to additive Gaussian noise, at moderate and high SNR levels. For low SNR levels the channel estimation performance is significantly degraded. Notice that, although the proposed order detection technique is based on the 4th-order output cumulants, the computation of $\hat{\rho}_{k}$ involves the estimation of the covariance matrix $\hat{\boldsymbol{\Sigma}}_{k}$, which requires computing 2 nd-order moments estimates. The overall detection performance is therefore expected to suffer with increasing levels of Gaussian noise, as illustrated in fig. 5. We notice that error propagation also affects the performance of the proposed method in a varying range of SNR.

Finally, we studied the case of two sources transmitting data over channels that share one common zero, with $L_{1}=2, L_{2}=1$ and $K=5$. In this case, the user channels have been generated by randomly choosing a point within a unit-sided square centered at the origin of the Real $\times$ Imaginary plane, following a bidimensional uniform distribution. The zero-pole plot of the channels used in this simulation is shown in the right-hand side of fig. 6. The left-hand side of fig. 6 shows NMSE versus the sample data length for $\mathrm{SNR}=40 \mathrm{~dB}$. Looking at these results, we notice a performance degradation with respect to the case shown in fig. 3. This observation suggests that the proposed method performs better with channels that 


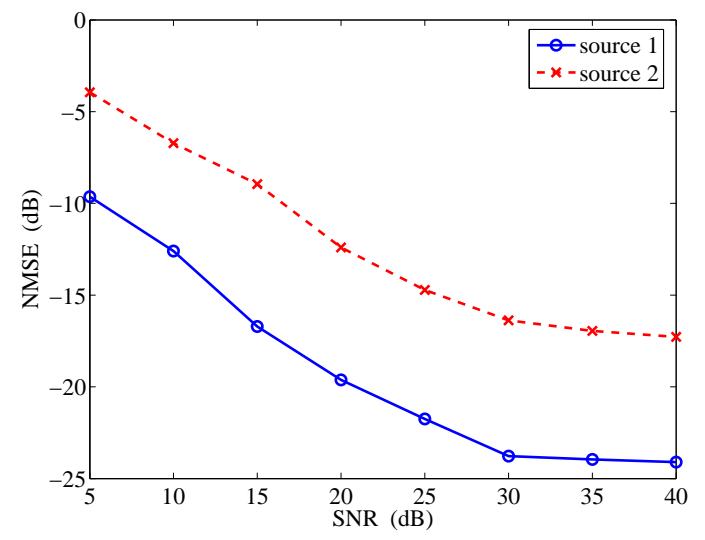

Figure 5: SNR $\times$ NMSE with $N=10^{4}$ symbols $\left(L_{1}=2, L_{2}=1\right.$ and $\left.K=5\right)$.
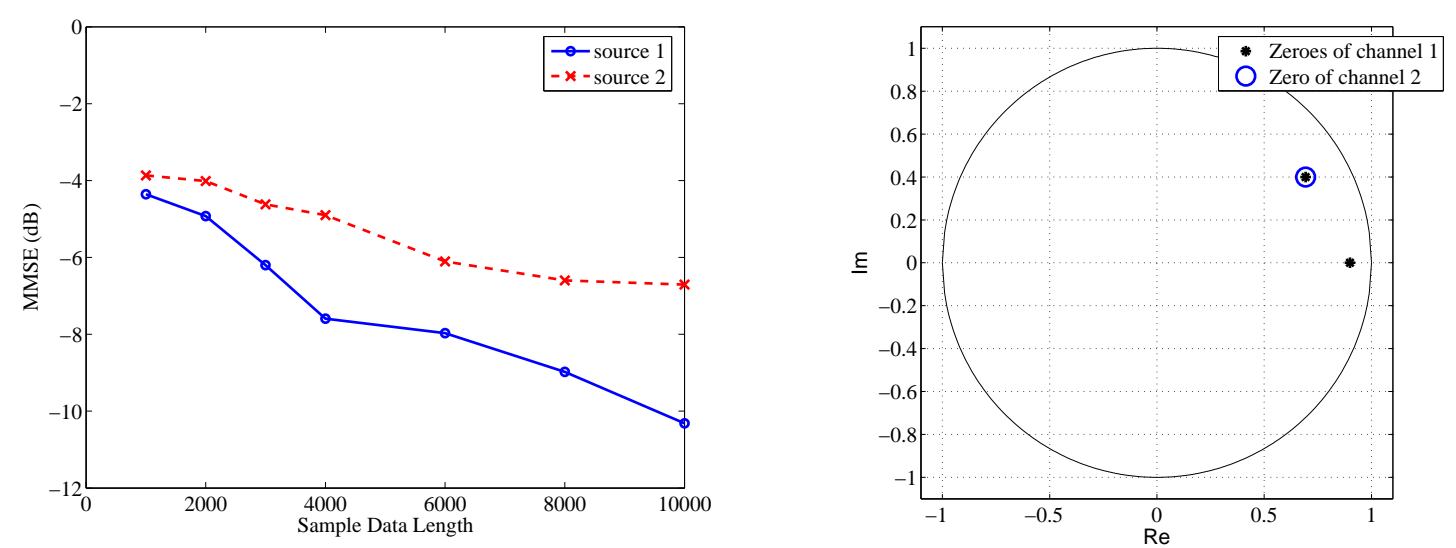

Figure 6: Left: NMSE versus the sample data length for $\mathrm{SNR}=40 \mathrm{~dB}$ with $L_{1}=2, L_{2}=1$ and $K=5$; Right: Zero-pole plot for channels 1 and 2.

do not share common zeroes.

\section{Conclusion}

In this paper, a new solution has been proposed to jointly solve the problems of order detection and parameter estimation of FIR channels in the context of a frequency-selective MISO communication system. We have proposed a channel order detection algorithm based on HOS hypothesis testing. A Chi-square test variable has been introduced along with a discussion about the choice of the decision threshold. The proposed detection algorithm relies on some properties of the 4th-order output cumulants. Exploiting the proposed order selection method, a combined detection-estimation procedure has been introduced. The new algorithm successively detects the source signals, determines the order of their individual transmission channel and estimates the associated channel coefficients. The nested detectors are based on a deflation approach, extracting from the output cumulants the marginal contributions of the previously identified channels and testing for the presence of shorter and shorter channels. Using the residual cumu- 
lant vector, the order-detection problem is treated separately for each user. Computer simulations have shown the good performance obtained for channel order detection and identification.

One main drawback of the proposed technique is the fact that it is based on a deflation technique, which entails a high sensitiveness to cumulant estimation errors, leading to cumulative parameter estimation errors. We currently workout a possible solution to mitigate or suppress the error propagation issue based on a adapted deflation approach for referenced contrast optimization 19].

The case of channels with the same order still needs further investigation. Future works on this subject include an implementation of the nested MISO detectors to the case of a multiple-input multipleoutput (MIMO) communication channel. In such a MIMO approach more information is available, which should allow us to treat the case of same length channels, including the overdetermined as well as the underdetermined mixtures.

\section{References}

[1] H. Akaike. A new look at the statistical model identication. IEEE Trans. on Automatic Control, 19(6):716723, 1974 .

[2] L. Albera and P. Comon. Asymptotic performance of contrast-based blind source separation algorithms. In 2nd IEEE Sensor Array and Multichannel Signal Processing Workshop, Rosslyn, USA, aug 2002.

[3] L. Albera, A. Ferréol, P. Comon, and P. Chevalier. Blind identification of overcomplete mixtures of sources (BIOME). Linear Algebra Applications, 391C:3-30, nov 2004.

[4] J. F. Böhme. Statistical array signal processing of measured sonar and seismic data. In Proc. SPIE Advanced Signal Processing Algorithms, San Diego, USA, jul. 1995.

[5] D. R. Brillinger. Time Series: Data analysis and theory. Holden-Day, San Francisco, 1981.

[6] D. R. Brillinger and M. Rosenblatt. Computation and interpretation of $k$ th-order spectra. In B. Harris, editor, Spectral analysis of time series, pages 189-232, New York, USA, 1967. Wiley.

[7] J.-F. Cardoso. Source separation using higher order moments. In Proc. of IEEE ICASSP, pages 2109-2112, Glasgow, Scotland, may. 1989.

[8] J.-F. Cardoso and A. Souloumiac. Blind beamforming for non gaussian signals. IEE Proceedings-F, 140(6):362-370, dec. 1993.

[9] P.-J. Chung, J. F. Böhme, C. F. Mecklenbräuker, and A. O. Hero. On signal detection using the BenjaminiHochberg procedure. In Proc. IEEE Workshop on Statistical Signal Processing, pages 615-618, Bordeaux, France, jul. 2005.

[10] P.-J. Chung, J. F. Böhme, C. F. Mecklenbräuker, and A. O. Hero. Detection of the number of signals using the Benjamini-Hochberg procedure. IEEE Trans. on Signal Processing, 55(6):2497-2508, jun. 2007.

[11] P.-J. Chung, N. Czink, and C. F. Mecklenbräuker. Model order selection for multipath MIMO channels using the Benjamini-Hochberg procedure. In ITG/IEEE-Workshop on Smart Antennas, Ulm, Germany, mar. 2006.

[12] P. Comon. Independent component analysis. In Proc. Int. Sig. Proc. Workshop on Higher-Order Statistics, pages 111-120, Chamrousse, France, July 1991.

[13] P. Comon. MA identification using fourth order cumulants. Signal Processing Elsevier, 26(3):381-388, mar. 1992. 
[14] P. Comon, P. Chevalier, and V. Capdevielle. Performance of contrast-based blind source separation. In Proc. SPAWC, pages 345-348, Paris, 1997.

[15] P. Comon and L. Deruaz. Normality tests for coloured samples. In Proc. IEEE-ATHOS Workshop on HOS, pages 217-221, Begur, Spain, jun. 1995.

[16] P. Comon and M. Rajih. Blind identification of under-determined mixtures based on the characteristic function. Signal Processing Elsevier, 86(9):2271-2281, sep 2006.

[17] L. De Lathauwer, B. De Moor, J. Vandewalle, and J.-F. Cardoso. Independent component analysis of largely underdetermined mixtures. In Proc. 4th Int. Symp. on Independent Component Analysis and Blind Signal Separation (ICA 2003), pages 29-34, Nara, Japan, apr. 2003.

[18] J.-P. Delmas, H. Gazzah, A. P. Liavas, and P. A. Regalia. Statistical analysis of some second-order methods for blind channel identification/equalization with respect to channel undermodeling. IEEE Trans. on Signal Processing, 48(7):1984-1998, Jul. 2000.

[19] R. Dubroca, C. de Luigi, and E. Moreau. Adapted deflation approach for referenced contrast optimization in blind MIMO convolutive source separation. In Proc. of ICA 2009, Paraty-RJ, Brazil, 2009. T. Adali et al. (eds.), LNCS 5441, pp. 243-250, Springer-Verlag Berlin Heidelberg 2009.

[20] C. E. R. Fernandes, P. Comon, and G. Favier. Order detection and blind identification of $2 \times 1$ MISO channels. In Proc. of IEEE ICASSP, vol. 3, pages 753-756, Honolulu, USA, apr. 2007.

[21] C. E. R. Fernandes, G. Favier, and J. C. M. Mota. Blind channel identification algorithms based on the Parafac decomposition of cumulant tensors: the single and multiuser cases. Signal Processing, Elsevier, 88(6):1382-1401, 2008.

[22] B. Friedlander and B. Porat. Asymptotically optimal estimation of MA and ARMA parameters of nongaussian processes from higher-order moments. IEEE Trans. on Automatic Control, 35(1):27-35, 1990.

[23] M. Hinich. Testing for Gaussianity and Linearity of a Stationary Time Series, Journal of Time Series Analysis, 3(3):169-176, 1982.

[24] N. L. Johnson, S. Kotz, and N. Balakrishnan. Continuous univariate distributions, vol. 1. Wiley, 1970.

[25] R. L. Kashyap. Inconsistency of the AIC rule for estimating the order of autoregressive models. IEEE Trans. on Automatic Control, AC-25(5):996-998, oct. 1980.

[26] M. Kaveh, H. Wang, and H. Hung. On the theoretical performance of a class of estimators of the number of narrow-band sources. IEEE Trans. on Acoustics, Speech and Signal Processing, 35(9):1350-1352, 1987.

[27] J.-L. Lacoume, P.-O. Amblard, and P. Comon. Statistiques d'ordre supérieur pour le traitement du signal. Masson, 1997. in French.

[28] A. P. Liavas, P. A. Regalia, and J.-P. Delmas. Blind channel approximation: Effective channel order determination. IEEE Trans. on Signal Processing, 47(12):3336-3344, dec. 1999.

[29] A. P. Liavas, P. A. Regalia, and J.-P. Delmas. Robustness of least-squares and subspace methods for blind channel identification/equalization with respect to effective channel undermodeling/overmodeling. IEEE Trans. on Signal Processing, 47(6):1636-1644, jun. 1999.

[30] W. S. Liggett Jr. Passive sonar: fitting models to multiple time series. In J. W. R. Griffiths and P. L. Stocklin, editors, Proc. of NATO ASI Signal Processing, Academic, pages 327-345, New York, USA, 1973. 
[31] P. Loubaton, E. Moulines, and P. Regalia. Signal processing advances in wireless and mobile communications, volume II - Trends in Single- and Multi-User Systems, chapter Subspace method for blind identification and deconvolution, pages 63-112. Prentice-Hall, 2000.

[32] J. W. Mauchly. Significance tests for sphericity of a Normal $n$-variate distribution. The Annals of Mathematical Statistics, 11(2):204-209, jun. 1940.

[33] K. A. Meraim, J.-F. Cardoso, A. Gorokhov, P. Loubaton, and E. Moulines. On subspace methods for blind identification of single-input/multiple-output FIR filters. IEEE Trans. on Signal Processing, 45(1):42-55, jan. 1997.

[34] K. A. Meraim, P. Loubaton, and E. Moulines. A subspace algorithm for certain blind identification problems. IEEE Trans. on Signal Processing, 43(2):499-511, mar. 1997.

[35] K. A. Meraim, E. Moulines, and P. Loubaton. Prediction error method for second order blind identification. IEEE Trans. on Signal Processing, 45(3):694-705, mar. 1997.

[36] E. Moulines, P. Duhamel, J.-F. Cardoso, and S. Mayrargue. Subspace methods for the blind identification of multichannel FIR filters. IEEE Trans. on Signal Processing, 43(2):516-525, feb. 1995.

[37] R. J. Muirhead. Aspects of multivariate statistical theory, volume (XIX-673 p.). John Wiley \& Sons, New York, 1982 .

[38] J. Neyman and E. S. Pearson. On the problem of the most efficient tests of statistical hypotheses. Philosophical Trans. of the Royal Society of London, A 231:289-337, 1933.

[39] B. Porat and B. Friedlander. Performance analysis of MA parameter estimation algorithms based on highorder moments. In Proc. of IEEE ICASSP, vol. 4, pages 2412-2415, New York City, USA, 1988.

[40] B. Porat and B. Friedlander. Performance analysis of parameter estimation algorithms based on high-order moments. Journal of Adaptive Control and Signal Processing, 3:191-229, 1989.

[41] B. Porat and B. Friedlander. Direction finding algorithms based on high-order statistics. IEEE Trans. on Signal Processing, 39(9):2016-2024, sep. 1991.

[42] J. Rissanen. Modeling by shortest data description length. Automatica, 14(5):465-471, 1978.

[43] G. Schwarz. Estimating the dimensions of a model. The Annals of Statistics, 6(2):461-464, 1978.

[44] P. Stoica and Y. Selén. Model order selection: a review of information criterion rules. IEEE Signal Processing Magazine, 21(4):36-47, 2004.

[45] L. Tong and S. Perreau. Multichannel blind identification: From subspace to maximum likelihood methods. Proceedings of the IEEE, 86:10, oct. 1998.

[46] L. Tong, G. Xu, and T. Kailath. Blind identication based on second-order statistics: A time domain approaches. IEEE Trans. on Information Theory, 40(2):340-349, 1994.

[47] H. L. Van Trees. Detection, estimation and modulation theory. Part I. Wiley, New York, 1968.

[48] M. Wax. Detection and estimation of superimposed signals. PhD thesis, Stanford University, Stanford, California, USA, mar. 1985.

[49] M. Wax and T. Kailath. Detection of signals by information theoretic criteria. IEEE Trans on Acoust., Speech and Signal Processing, ASSP-33:387-392, apr. 1985.

[50] D. B. Williams and D. H. Johnson. Modifying the sphericity test for improved source detection with narrowband passive arrays. In Proc. of IEEE ICASSP, vol. 12, pages 2272-2275, Dallas, USA, apr. 1987. 
[51] D. B. Williams and D. H. Johnson. Using the sphericity test for source detection with narrow-band passive arrays. IEEE Trans. on Signal Processing, 38(11):2008-2014, nov. 1990.

[52] Y. Yu and A.P. Petropulu. Robust PARAFAC based blind estimation of MIMO systems with possibly more inputs than outputs. In Proc. of IEEE ICASSP, pages 133-136, Toulouse, France, may 2006. 